\title{
Characterization of Virologic Failure After an Initially Successful 48-Week Course of Antiretroviral Therapy in HIV/AIDS Outpatients Treated in Santos, Brazil
}

\author{
Marcos M. Caseiro', Alcino A.C. Golegã ${ }^{1}$, Arnaldo Etzel ${ }^{1}$ and Ricardo Sobhie Diaz ${ }^{2}$ \\ ${ }^{1}$ Fundação Lusiadas, Santos, SP; ${ }^{2}$ Paulista School of Medicine, Federal University of São Paulo; São Paulo, SP, Brazil
}

\begin{abstract}
We characterized the virologic failure after an initially successful 48-week course of antiretroviral therapy among HIV/AIDS patients in a retrospective cohort study involving patients from Santos, Brazil. Patients with plasma HIV RNA below 500 copies/mL for 48 weeks were included. Variables analyzed included gender, age, level of education, marital status, mode of HIV acquisition, viral load, and CD4 cell count upon admission. There were 4,909 patients registered with the clinic, of which 669 patients met all the inclusion criteria ( $41.6 \%$ female and $58.4 \%$ male). Only $27.5 \%$ of the patients maintained undetectable viral loads during up to one year of follow-up. After 48 weeks, virologic failure occurred earlier in females and in patients first treated with an antiretroviral regimen other than highly active antiretroviral therapy. Patients who were married or had a steady partner experienced virologic failure later than did those who were separated or widowed. The percentage of public health clinic patients who maintain undetectable viral loads for a period of over a year is much lower than that observed among patients enrolled in clinical trials. Females, individuals in unstable relationships, single individuals and widowed individuals should be given special attention in order to improve durability of viral suppression.

Key-Words: Antiretroviral therapy, virologic failure, HIV, Brazil.
\end{abstract}

The treatment of HIV-positive patients with highly active antiretroviral therapy (HAART), has resulted in a dramatic decrease in AIDS-related morbidity and mortality, as has been demonstrated by various authors [1-4]. Previous studies indicate that this combined therapy decreases plasma levels of HIV RNA, as seen in two separate studies reporting levels of less than 50 copies $/ \mathrm{mm}^{3}$ in $60 \%$ of patients and less than 500 copies $/ \mathrm{mm}^{3}$ in $90 \%$ of patients [5-7]. However, since antiretroviral therapy involves regimens that are often complex and present side effects in a great number of patients, it is difficult to achieve a high degree of adherence to treatment, which is required in order to maintain viral suppression $[5,6,8]$. Taking these difficulties into consideration, it is clear that the success of HAART regimens depends on the quality of health services. In addition, these regimens might be less efficient, in various forms, at specialized nonuniversity clinical outpatient facilities that do not engage in clinical trials [9]. In addition, in contrast with developed countries where HAART is available to HIV-positive patients, this therapy is unavailable in most developing countries, where over $90 \%$ of these patients reside [10]. Before the HAART era, various studies revealed a lower survival rate among HIV-positive ethnic minorities, women, intravenous drug users and individuals of low socioeconomic status [11-13]. Some studies, carried out after the advent of HAART, have clearly shown that the sustained viral suppression rate is lower in outpatients evaluated in a large urban clinic than in those monitored in clinical trials [10]. The

Received on 11 December 2007; revised 14 April 2008.

Address for correspondence: Dr. Ricardo S. Diaz. Laboratório de Retrovirologia. R. Pedro de Toledo, 781, São Paulo, SP, Brazil. Zip code: 04039-032. Phone: (55) (11) 9109-0445; Fax: (55 11) 41923176. E-mail: rsodiaz@terra.com.br.

The Brazilian Journal of Infectious Diseases 2008;12(3):162-166. (C) 2008 by The Brazilian Journal of Infectious Diseases and Contexto Publishing. All rights reserved. effect that demographic differences have on the success of HAART is less evident.

Santos is a coastal city located in the south of the state of Sao Paulo, Brazil, and has a population of 440,000 inhabitants. Santos is a major tourist destination and the largest port in Latin America. People from various regions of Brazil and the rest of the world visit the city. Since the beginning of the AIDS epidemic in Brazil, Santos has been known as a city with one of the highest AIDS prevalence rates.

Santos was the first city in the country to provide free protease inhibitors (PIs) to its patients and has been distributing them without restriction since February of 1996. Subsequently, the National STD/AIDS Program of the Brazilian Ministry of Health began to provide universal access to all HAART medications $[14,15]$. This policy has resulted in significantly lower AIDS-related mortality and morbidity 15 as seen in other countries [1,4]. Comprehensive studies of the characteristics related to antiretroviral therapy adherence have been carried out in Brazil. However, some of those studies did not employ objective markers of the evolution of the disease, such as CD4 and CD8 T-lymphocyte counts and HIV RNA plasma levels, to evaluate the consequent therapeutic response $[16,17]$. In the current study we attempted to characterize the virologic failure after an initial 48-week virologic success among patients on antiretroviral therapy in the city of Santos.

\section{Material and Methods}

This retrospective cohort study involved monitored patients registered with the Santos Referral Center for AIDS (CRAIDS), in order to assess the occurrence of virologic failure in individuals undergoing antiretroviral treatment. CRAIDS is the only outpatient clinic for adults with HIV/ AIDS in the city of Santos. The study sample comprised patients treated with any type of antiretroviral regimen between 1997 and 2003. Those whose plasma HIV RNA levels remained below 500 copies/mL for at least 48 weeks were 
included. Data from routine laboratory tests required in the clinic were used in this study, according to the instructions from the Ministry of Health that recommends virologic testing every four months. The criterion used in the definition of virologic failure was plasma HIV RNA higher than 500 copies/ $\mathrm{mL}$ after inclusion in the study. Patient's data were collected up to December 31, 2003. Sociodemographic variables (gender, age bracket, level of education and marital status), behavioral variables (mode of HIV acquisition) and markers of evolution (viral load and number of CD4 cells upon admission) were included as possible explanatory variables for virologic failure. The antiretroviral therapy was analyzed in a comprehensive way (HAART and non-HAART), with no distinctions being made between specific regimens. Data regarding adherence to treatment were also not considered. Data collection was carried out through the review of patient medical charts on file at CRAIDS. The information was initially registered on a pre-structured questionnaire. The statistical analysis was performed by entering data into a database constructed using the Epi Info program, version 3.3.0. Initially, we carried out a descriptive analysis of the general characteristics of the population studied regarding the occurrence of virologic failure and exposure to the explanatory variables proposed. The quantitative and qualitative variables were categorized using interpretative criteria based on biological or epidemiological rationale. Survival curves were calculated using the Kaplan-Meier product-limit estimate in order to determine the general survival function, and the curves were constructed according to the inclusion stratum in the study of the explanatory variables considered. In this phase, we observed whether the curves overlapped. If they did not overlap, they would comply with the visual assumptions of proportionality over time. The different variables were not adjusted to one another and were compared using the logrank test, observed for each stratum of the respective variables. CD4 and CD8 T-lymphocyte subpopulation counting was performed by flow cytometry using the FACSCount system (Becton Dickinson, San Jose, CA, USA). HIV-1 viral load was performed using the NASBA technique (Biomerieux, Marcy l'Etoile, France). The laboratory belongs to the Santos Municipal Secretary of Health and has an operating agreement with the Brazilian Ministry of Health, which is responsible for quality control.

\section{Results}

There were 4,909 patients registered with the clinic, of which 669 patients remained with HIV RNA viral load below 500 copies/mL for at least 48 weeks. This represented the 27.5\% of all CRAIDS patients undergoing antiretroviral therapy and presenting viral loads that remained lower than 500 copies/mL for over 48 weeks.

Of the 669 patients studied, 278 (41.6\%) were female and 381 (58.4\%) were male (Table 1 ). The mean age was 42 years, and the majority of patients fell into one of two age brackets: 21 to 40 (47.7\%); and 41 to 60 (46.9\%) (Table 1$)$. A total of 233 patients (34.8\%) were married. The most common mode of exposure to HIV, regardless of gender, was through unprotected sex with multiple partners or with a partner who had multiple partners, accounting for 421 cases (62.9\%). In general, females presented lower levels of education, although education was found not to be related to virologic failure. A total of $52.9 \%$ of females, in contrast to $43.5 \%$ of males, had either no schooling or less than 8 years of schooling. In addition, only $28.4 \%$ of females had completed high school, compared with $35.3 \%$ of males. However, there was no significant difference between males and females in terms of higher education. All of these data, grouped by gender, can be observed in Table 1.

Baseline CD4 counts revealed 0-200 cells/mm3 in 229 patients (34.3\%); 201-350 cells $/ \mathrm{mm}^{3}$ in 155 (23.2\%); 351-500 cells/ $\mathrm{mm}^{3}$ in 112 (16.8\%); and $>500$ cells $/ \mathrm{mm}^{3}$ in 171 (25.6\%). Baseline viral loads were lower than 10,000 copies $/ \mathrm{mm}^{3}$ in 461 (68.9\%) of the patients; between 10,001 and 100,000 copies/ $\mathrm{mm}^{3}$ in 139 (20.8\%); and greater than 100,001 copies $/ \mathrm{mm}^{3}$ in 69 (10.3\%).

Initial antiretroviral regimens used by the patients were as follows: 1 NRTI, by $10.4 \%$ (29) of the females and 3.6\% (14) of the males; 2 NRTIs, $36.7 \%$ (102) of the females and $29.2 \%$ (114) of the males; 3 NNRTIs, $1.4 \%$ (4) of the females and $1.0 \%$ (4) of the males; 2 NRTIs plus 1 NNRTI, 26.3\% (73) of the females and 25.6\% (100) of the males; 2 NRTI plus 1 PI, 25.2\% (70) of the females and $40.7 \%$ (159) of the males. When we grouped patients by the type of antiretroviral therapy used, we observed that 404 (60.4\%) of the patients used HAART. The 265 (39.6\%) that used non-HAART drugs, which is not recommended by current guidelines, nevertheless maintained viral loads below 500 copies $/ \mathrm{mm}^{3}$ for over twelve months.

Using the Kaplan-Meier test to analyze the durability of viral suppression, defined as an undetectable viral load, after an initially successful 48-week course of antiretroviral therapy, we observed that virologic failure occurred earlier in females than in males (Figure 1).

Using the same test to evaluate only those patients initially receiving HAART, we observed that a smaller difference between female/male regarding the occurrence of virologic failure, with a tendency towards equality between 60 and 70 months of follow-up (Figure 2).

In our study sample, the baseline CD4 values and viral load did not present Kaplan-Meier correlations with the durability of viral suppression.

We found that patients who were first treated with 1 NRTI, 2 NRTIs or 3 NRTIs presented less viral suppression durability over time than did those who were first treated with 2 NRTIs + 1 NNRTI or with 1 PI (Figure 3).

The only sociodemographic variable found to affect the duration of viral suppression was being married or having a steady partner. Among patients for whom the initial therapy was HAART, those who were married or had a steady partner experienced virologic failure later than did those who were separated or widowed (Figure 4). 
Table 1. Sociodemographic profile of the patients in relation to the ocurrence of virologic failure.

\begin{tabular}{|c|c|c|c|c|c|c|}
\hline \multirow{2}{*}{$\frac{\text { Variable }}{\text { Age }}$} & \multicolumn{2}{|c|}{ Female } & \multicolumn{2}{|c|}{ Male } & \multirow[t]{2}{*}{ OR } & \multirow[t]{2}{*}{$\mathbf{p}$} \\
\hline & & & & & & \\
\hline 21 to 40 years & $52.9 \%$ & 147 & $44.0 \%$ & 172 & NS & NS \\
\hline 41 to 60 years & $40.6 \%$ & 113 & $51.4 \%$ & 201 & 1.52 & $<0.0001$ \\
\hline$>60$ years & $6.5 \%$ & 18 & $4.6 \%$ & 18 & 0.85 & 0.65 \\
\hline \multicolumn{7}{|l|}{ Education } \\
\hline Illiterate/literate & $5.8 \%$ & 16 & $2.6 \%$ & 10 & NS & NS \\
\hline Elementary school & $47.1 \%$ & 131 & $43.5 \%$ & 291 & 3.55 & $<0.001$ \\
\hline High school & $28.4 \%$ & 79 & $35.3 \%$ & 138 & 2.79 & 0.01 \\
\hline Higher education & $10.1 \%$ & 28 & 13.3 & 52 & 2.97 & 0.01 \\
\hline Without information & $8.6 \%$ & 24 & 7.9 & 31 & NS & NS \\
\hline \multicolumn{7}{|l|}{ Marital status } \\
\hline Married/cohabiting & $37.8 \%$ & 105 & $32.7 \%$ & 128 & NS & NS \\
\hline Widowed/divorced & $28.8 \%$ & 80 & $17.4 \%$ & 68 & 0.7 & 0.08 \\
\hline Single & $25.2 \%$ & 70 & $39.1 \%$ & 153 & 1.79 & 0.002 \\
\hline Other & $5.0 \%$ & 14 & $7.9 \%$ & 31 & 1.28 & 0.08 \\
\hline Without information & $3.2 \%$ & 9 & $2.8 \%$ & 11 & NS & NS \\
\hline \multicolumn{7}{|l|}{ Type of contamination } \\
\hline Injectable drugs & $4.3 \%$ & 12 & $7.4 \%$ & 29 & - & \\
\hline Multiple partners & $25.5 \%$ & 71 & $35.8 \%$ & 140 & NS & NS \\
\hline Individual with multiple partners & $27.3 \%$ & 76 & $34.3 \%$ & 134 & NS & NS \\
\hline Partner HIV/AIDS & $37.4 \%$ & 104 & $17.4 \%$ & 68 & 0.27 & $>0.0001$ \\
\hline Not known & $5.4 \%$ & 15 & $5.1 \%$ & 20 & NS & NS \\
\hline \multicolumn{7}{|l|}{ Initial CD4 (First) } \\
\hline 0 to 200 & $27.5 \%$ & 76 & $39.1 \%$ & 153 & NS & NS \\
\hline 201 to 350 & $20.7 \%$ & 57 & $25.1 \%$ & 98 & NS & NS \\
\hline 351 to 500 & $21.4 \%$ & 59 & $13.6 \%$ & 53 & NS & NS \\
\hline$>500$ & $30.4 \%$ & 84 & $22.3 \%$ & 87 & NS & NS \\
\hline \multicolumn{7}{|l|}{ Initial viral load (First) } \\
\hline 0 to 10,000 & $70.1 \%$ & 195 & $68.0 \%$ & 266 & NS & NS \\
\hline 10,001 to 100,000 & $20.1 \%$ & 56 & $21.2 \%$ & 83 & NS & NS \\
\hline 100,001 to $1,000,000$ & $9.7 \%$ & 27 & $10.7 \%$ & 42 & NS & NS \\
\hline \multicolumn{7}{|l|}{ Initial medication } \\
\hline Antiretroviral therapy & $48.2 \%$ & 134 & $33.5 \%$ & 131 & - & - \\
\hline HAART & $51.8 \%$ & 144 & $66.5 \%$ & 260 & 1.85 & $>0.0001$ \\
\hline Total & $\%$ & $(n=278)$ & $\%$ & $(n=391)$ & & \\
\hline
\end{tabular}

OR=odds ratio; $\mathrm{p}=\mathrm{p}$ value; $\mathrm{NS}=$ not significant.

Figure 1. Duration of viral suppression, by gender, as estimated using the Kaplan-Meier test, in 669 HIV-positive patients treated at the Santos Referral Center for AIDS between 1997 and 2003.

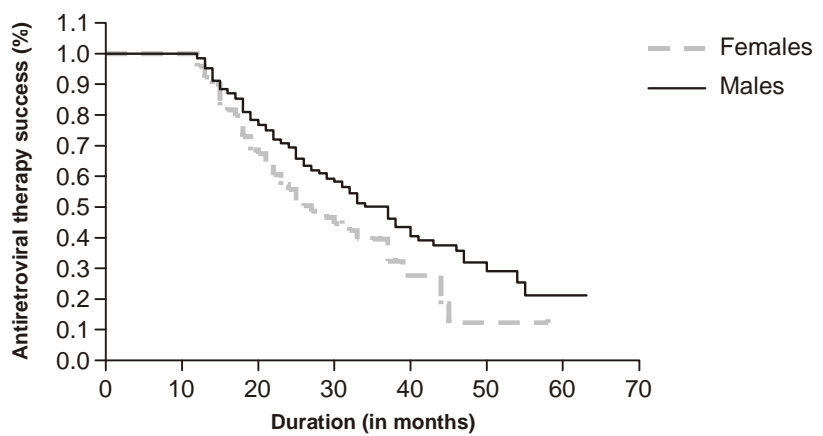

Figure 2. Kaplan-Meier test: duration of viral suppression prior to virologic failure, by gender, among patients using $\operatorname{HAART}(\mathrm{n}=404)$.

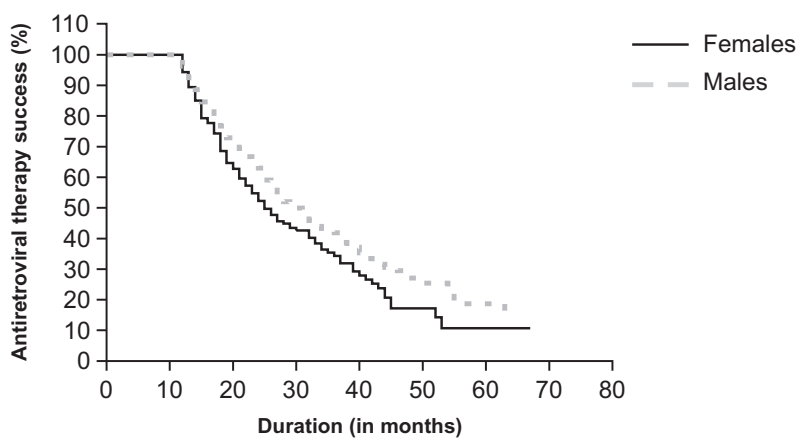


Figure 3. Kaplan-Meier test: duration of viral suppression prior to virologic failure, by type of antiretroviral therapy initially used ( $\mathrm{n}=669)$.

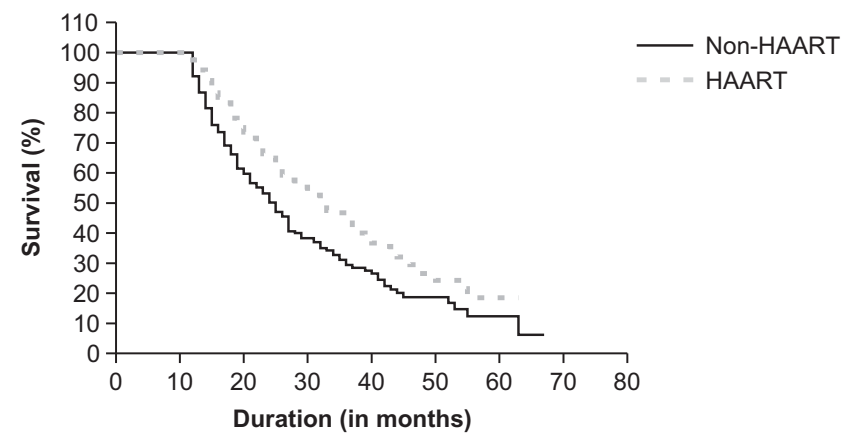

\section{Discussion}

In this retrospective analysis of the effectiveness of HAART in patients treated at public health clinics, we found that the proportion of such patients in whom therapeutic success was achieved was much lower than that found in patients treated in clinical trials $[5,18,19]$. However, the results are similar to the findings reported for public and private urban clinics in the USA [2], Europe [9] and Brazil [20,21].

In 2004, a study conducted in Brazil involving 211 patients in 5 public health clinics in Rio de Janeiro, revealed that $44 \%$ of these patients achieved undetectable viral loads within 6 months [20]. However, there are no sequential data regarding the maintenance of these values. Additional data reported that the median time to virologic failure was approximately 14 months among Brazilian treatment-naïve patients [21], and presumably, antiretroviral treatment failure rates are higher among those receiving sequential treatment.

In the current study, we detected that only $27.5 \%$ of the patients maintained viral loads below detection limits for one year. It is of note that these data represent patients who achieved this objective with the first antiretroviral therapy administered. This fact is certainly extremely relevant and alarming, since a high risk of failure is expected from these patients over time, in addition to the high rate of primary resistance recently described for the city of Santos, which is approximately 36\% among patients with recent infection, and the high rates of non-B clade, which is around 50\% [22].

Concerning other factors related to virologic failure, we observed that being a female, being single and being widowed were related to higher rates of failure. In a comprehensive study of adherence carried out in Brazil $[16,17]$, gender-related alterations in adherence were not observed, although marital status was not assessed in those studies. We find our observation extremely important, since it places females in a situation of greater vulnerability, which might have been overlooked in the surveys conducted by other authors. Taking into consideration the fact that, although the levels of HIV-1 RNA are usually lower in women than in men, the rates of progression to AIDS are similar between men and women [23],
Figure 4. Kaplan-Meier test: duration of viral suppression prior to virologic failure, by marital status, among the group of patients receiving HAART.

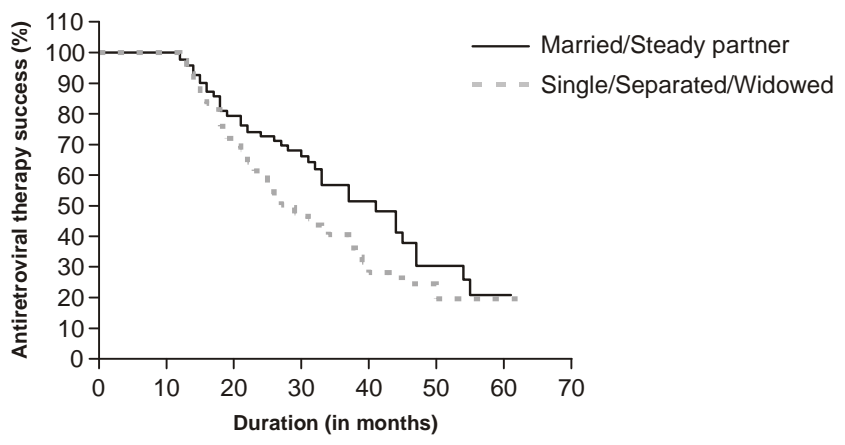

in addition to the theoretical possibility of pregnancy, we believe this observation to be highly relevant. Furthermore, it has been suggested that females are at a higher risk of presenting adverse effects [9], a fact that was not confirmed in the current study. It is interesting to note that there was a trend for women to be treated more frequently without HAART, with fewer PIs, and that the difference in virologic response among men and women is not present when HAART is used. Other studies are necessary in order to provide more accurate information that would confirm the tendency of higher virologic failure among women. We found that patients in stable marital relationships maintain undetectable viral loads for a longer period of time, probably reflecting more propitious conditions for the use of this medication in patients with stable family relationships. We did not observe any difference among individuals of different educational levels in terms of viral suppression, a finding that is in contrast with those of other studies conducted in Brazil [21]. This suggests that despite the fact that, in Brazil, HIV tends to affect individuals of lower socioeconomic status, antiretroviral therapy can be used appropriately, provided these patients are properly instructed and motivated. It is important to realize that, in contrast to the findings of other studies [21], viral suppression, in our study sample, was not found to be influenced by the CD4 nadir. This probably reflects the potency of the new antiretroviral regimens and their introduction in the period prior to the analysis of these data. Finally and not surprisingly, the use of HAART, understood here as the triple therapy with 2 NRTIs plus 1 NNRTI or with 1 PI, was associated with greater viral suppression durability after the initial 48 weeks of viral suppression, to the detriment of other regimens that are unfortunately still in use, such as the double-NRTI or tripleNRTI regimens. This finding suggests that even in the initial presence of viral suppression with less potent regimes, it may be worth increasing the potency of the antiretroviral scheme in order to prevent an earlier virologic failure.

We acknowledge that the retrospective nature of this study may prevent more definitive conclusions, especially due to the lack of information on adherence to treatment. However 
we believe that the design of future prospective studies may take into account the information presented here.

In conclusion, our data suggest that the percentage of public health clinic patients who maintain undetectable viral loads for a period of over a year is much lower than that observed among patients enrolled in clinical trials. However, it is similar to those observed among patients treated at other urban clinics. Females, individuals in unstable relationships, single individuals and widowed individuals should be given special attention in order to improve and make the antiretroviral therapy more appropriate, thus increasing the durability of viral suppression. Further studies should be conducted in order to better understand the differences observed between various locations, so that effective measures can be taken to adapt the therapy in order to improve and prolong antiretroviral response, consequently decreasing morbidity and mortality.

\section{References}

1. Palella F.J., Jr., Delaney K.M., Moorman A.C., et al. Declining morbidity and mortality among patients with advanced human immunodeficiency virus infection. HIV Outpatient Study Investigators. N Engl J Med 1998;338(13):853-60.

2. Holmberg S.D., Hamburger M.E., Moorman A.C., et al. Factors associated with maintenance of long-term plasma human immunodeficiency virus RNA suppression. Clin Infect Dis 2003;37(5):702-7.

3. Kleeberger C.A., Buechner J., Palella F., et al. Changes in adherence to highly active antiretroviral therapy medications in the Multicenter AIDS Cohort Study. AIDS 2004;18(4):683-8.

4. Detels R., Munoz A., McFarlane G., et al. Effectiveness of potent antiretroviral therapy on time to AIDS and death in men with known HIV infection duration. Multicenter AIDS Cohort Study Investigators. JAMA 1998;280(17):1497-503.

5. Flexner C. HIV-protease inhibitors. N Engl J Med Apr 30 1998;338(18):1281-92.

6. Deeks S.G., Smith M., Holodniy M., Kahn J.O. HIV-1 protease inhibitors. A review for clinicians. JAMA 1997;277(2):145-53.

7. Tierney C., Lathey J.L., Christopherson C., et al. Prognostic value of baseline human immunodeficiency virus type 1 DNA measurement for disease progression in patients receiving nucleoside therapy. J Infect Dis 2003;187(1):144-8.

8. Vanhove G.F., Schapiro J.M., Winters M.A., et al. Patient compliance and drug failure in protease inhibitor monotherapy. JAMA 1996;276(24):1955-6.
9. Lucas G.M., Chaisson R.E., Moore R.D. Highly active antiretroviral therapy in a large urban clinic: risk factors for virologic failure and adverse drug reactions. Ann Intern Med. Jul 20 1999;131(2):81-87.

10. Piot P. Global AIDS epidemic: time to turn the tide. Science 2000;288(5474):2176-8.

11. Friedland G.H., Saltzman B., Vileno J., et al. Survival differences in patients with AIDS. J Acquir Immune Defic Syndr 1991;4(2):14453.

12. Rothenberg R., Woelfel M., Stoneburner R., et al. Survival with the acquired immunodeficiency syndrome. Experience with 5833 cases in New York City. N Engl J Med 1987;317(21):1297-302.

13. Hogg R.S., Strathdee S.A., Craib K.J., et al. Lower socioeconomic status and shorter survival following HIV infection. Lancet 1994;344(8930):1120-4.

14. Galvao J. Access to antiretroviral drugs in Brazil. Lancet 2002;360(9348):1862-5.

15. Levi G.C., Vitoria M.A. Fighting against AIDS: the Brazilian experience. AIDS 2002;16(18):2373-83.

16. Nemes M.I., Carvalho H.B., Souza M.F. Antiretroviral therapy adherence in Brazil. AIDS 2004;18 Suppl 3:S15-20.

17. Nemes M.I., Castanheira E.R., Melchior R., et al. [Evaluating quality of care in an AIDS program: health services research issues in Brazil]. Cad Saude Publica 2004;20 Suppl 2:S310-21.

18. Hammer S.M., Squires K.E., Hughes M.D., et al. A controlled trial of two nucleoside analogues plus indinavir in persons with human immunodeficiency virus infection and CD4 cell counts of 200 per cubic millimeter or less. AIDS Clinical Trials Group 320 Study Team. N Engl J Med 1997;337(11):725-33.

19. Hoesley C.J., Saag M.S., Chatham A., Kilby J.M. Prolonged suppression of human immunodeficiency virus type 1 RNA during dual nucleoside reverse-transcriptase-inhibitor therapy in clinical practice. Clin Infect Dis 2000;31(4):1095-7.

20. Hofer C.B., Schechter M., Harrison L.H. Effectiveness of antiretroviral therapy among patients who attend public HIV clinics in Rio de Janeiro, Brazil. J Acquir Immune Defic Syndr 2004;36(4):967-71.

21. Medeiros R., Diaz R.S., Filho A.C. Estimating the length of the first antiretroviral therapy regiment durability in Sao Paulo, Brazil. Braz J Infect Dis 2002;6(6):298-304.

22. Sucupira M.C., Caseiro M.M., Alves K., et al. High levels of primary antiretroviral resistance genotypic mutations and $\mathrm{B} / \mathrm{F}$ recombinants in Santos, Brazil. AIDS Patient Care STDS 2007;21(2):116-28.

23. Sterling T.R., Vlahov D., Astemborski J., et al. Initial plasma HIV1 RNA levels and progression to AIDS in women and men. N Engl J Med 2001;344(10):720-5. 